\title{
BLUE BRAIN
}

\author{
Chandani R. Suryawanshi ${ }^{1}$, Vinod Nayyar ${ }^{2}$ \\ Dept. of Wireless Communication \& Computing, Abha Gaikawad Patil College Of Engg. \\ Nagpur, INDIA \\ ${ }^{1}$ Krunal386@gmail.com, \\ vinodnayyar5@gmail.com
}

\begin{abstract}
Today scientists are in research to create an artificial brain that can think, respond, take decision, and keep anything in memory. The main aim is to upload human brain into machine. So that man can think, take decision without any effort. After the death of the body, the virtual brain will act as the man. So, even after the death of a person we will not loose the knowledge, intelligence, personalities, feelings and memories of that man, that can be used for the development of the human society. Technology is growing faster than every thing. IBM is now in research to create a virtual brain, called "Blue brain". If possible, this would be the first virtual brain of the world. IBM, in partnership with scientists at Switzerland's Ecole Polytech- nique Federale de Lausanne's (EPFL) Brain and Mind Institute will begin simulating the brain's biological systems and output the data as a working 3-dimensional model that will recreate the high-speed electrochemical interactions that take place within the brain's interior. These include cognitive functions such as language, learning, perception and memory in addition to brain malfunction such as psychiatric disorders like depression and autism. From there, the modeling will expand to other regions of the brain and, if successful, shed light on the relationships between genetic, molecular and cognitive functions of the brain.
\end{abstract}

\section{KEYWORDS}

FTP (floting point unit); ASIC (application specific integrated circuit)

\section{Council for Innovative Research}

Peer Review Research Publishing System

Journal: International Journal of Management \& Information Technology

Vol. 7, No. 2

editor@cirworld.com

www.cirworld.com, member.cirworld.com 


\section{INTRODUCTION}

Human brain is the most valuable creation of God. The man is called intelligent because of the brain. The brain translates the information delivered by the impulses, which then enables the person to react. But we loss the knowledge of a brain when the body is destroyed after the death of man. That knowledge might have been used for the development of the human society. What happen if we create a brain and up load the contents of natural brain into it?

\subsection{Blue Brain}

The name of the world's first virtual brain. That means a machine that can function as human brain. Today scientists are in research to create an artificial brain that can think, response, take decision, and keep anything in memory. The main aim is to upload human brain into machine. So that man can think, take decision without any effort. After the death of the body, the virtual brain will act as the man .So, even after the death of a person we will not loose the knowledge, intelligence, personalities, feelings and memories of that man that can be used for the development of the human society. No one has ever understood the complexity of human brain. It is complex than any circuitry in the world. So, question may arise "Is it really possible to create a human brain?" The answer is "Yes". Because what ever man has created today always he has followed the nature. When man does not have a device called computer, it was a big question for all. Technology is growing faster than every thing. IBM is now in research to create a virtual brain, called "Blue brain". If possible, this would be the first virtual brain of the world. With in 30 years, we will be able to scan ourselves into the computers. Is this the beginning of eternal life?

\subsection{What is Virtual Brain?}

Virtual brain is an artificial brain, which does not actually the natural brain, but can act as the brain. It can think like brain, take decisions based on the past experience, and response as the natural brain can. It is possible by using a super computer, with a huge amount of storage capacity, processing power and an interface between the human brain and this artificial one. Through this interface the data stored in the natural brain can be up loaded into the computer. So the brain and the knowledge, intelligence of anyone can be kept and used for ever, even after the death of the person.

\subsection{Why we need Virtual Brain?}

Today we are developed because of our intelligence. Intelligence is the inborn quality that can not be created. Some people have this quality, so that they can think up to such an extent where other can not reach. Human society is always need of such intelligence and such an intelligent brain to have with. But the intelligence is lost along with the body after the death. The virtual brain is a solution to it. The brain and intelli- gence will alive even after the death. We often face difficulties in remembering things such as people's names, their birthdays, and the spellings of words, proper grammar, important dates, history, facts etc... In the busy life every one want to be relaxed. Can't we use any machine to assist for all these? Virtual brain may be the solution to it. What if we upload ourselves into computer, we were simply aware of a computer, or maybe, what if we lived in a computer as a program?

\subsection{How it is possible?}

First, it is helpful to describe the basic manners in which a person may be uploaded into a computer. Raymond Kurzweil recently provided an interesting paper on this topic. In it, he describes both invasive and noninvasive techniques. The most promising is the use of very small robots, or nanobots. These robots will be small enough to travel throughout our circulatory systems. Traveling into the spine and brain, they will be able to monitor the activity and structure of our central nervous system.

They will be able to provide an interface with computers that is as close as our mind can be while we still reside in our biological form. Nanobots could also carefully scan the structure of our brain, providing a complete readout of the connections between each neuron. They would also record the current state of the brain. This information, when entered into a computer, could then continue to function as us. All that is required is a computer with large enough storage space and processing power. Is the pattern and state of neuron connections in our brain truly all that makes up our conscious selves? Many people believe firmly those we posses a soul, while some very technical people believe that quantum forces contribute to our awareness. But we have to now think technically. Note, however, that we need not know how the brain actually functions, to transfer it to a computer. We need only know the media and contents. The actual mystery of how we achieved consciousness in the first place, or how we maintain it, is a separate discussion. Really this concept appears to be very difficult and complex to us. For this we have to first know how the human brain actually works.

\section{HOW THE BLUE BRAIN PROJECT WILL WORK?}

\subsection{Goals \& Objectives}


The Blue Brain Project is the first comprehensive attempt to reverse-engineer the mammalian brain, in order to understand brain function and dysfunction through detailed simulations. The mission in undertaking The Blue Brain Project is to gather all existing knowledge of the brain, accelerate the global research effort of reverse engineering the structure and function of the components of the brain, and to build a complete theoretical framework that can orchestrate the reconstruction of the brain of mammals and man from the genetic to the whole brain levels, into computer models for simulation, visualization and automatic knowledge archiving by 2015. Biologi- cally accurate computer models of mammalian and human brains could provide a new foundation for understanding functions and malfunctions of the brain and for a new generation of information-based, customized medicine.

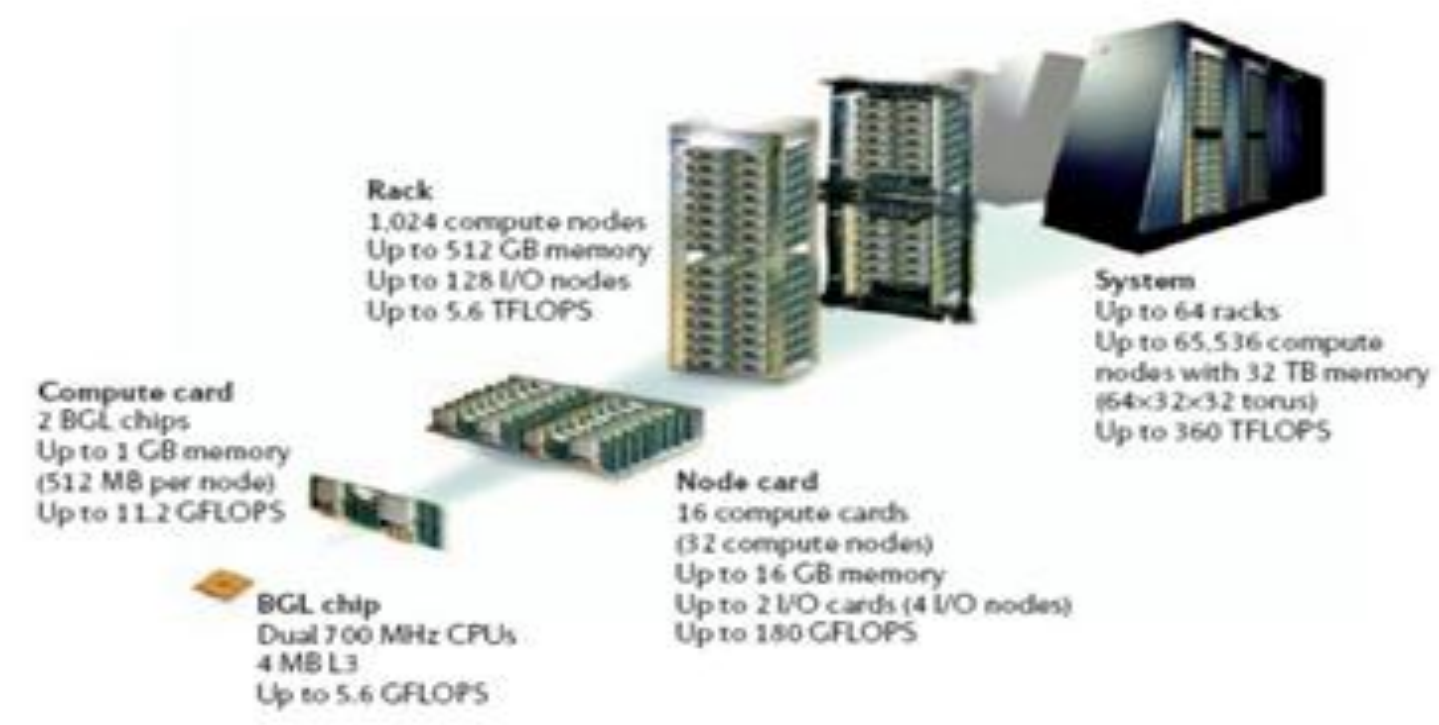

Fig. 1.1. The Blue Gene/L supercomputer architecture

\subsection{Architecture of Blue Gene}

Blue Gene/L is built using system-on-a-chip technology in which all functions of a node (except for main memory) are integrated onto a single application-specific integrated circuit (ASIC). This ASIC includes 2 PowerPC 440 cores running at $700 \mathrm{MHz}$. Associated with each core is a 64-bit "double" floating point unit (FPU) that can operate in single instruction, multiple data (SIMD) mode. Each (single) FPU can execute up to 2 "multiply-adds" per cycle, which means that the peak performance of the chip is 8 floating point operations per cycle (4 under normal conditions, with no use of SIMD mode). This leads to a peak performance of 5.6 billion floating point operations per second (gigaFLOPS or GFLOPS) per chip or node, or 2.8 GFLOPS in non- SIMD mode. The two CPUs (central processing units) can be used in "co- processor" mode (resulting in one CPU and 512 MB RAM (random access memory) for computation, the other CPU being used for processing the $\mathrm{I} / \mathrm{O}$ (input/output) of the main CPU) or in "virtual node" mode (in which both CPU swith $256 \mathrm{MB}$ each are used for computation). So, the aggregate performance of a processor card in virtual node mode is : $2 \times$ node $=2 \times 2.8$ GFLOPS = 5.6 GFLOPS, and its peak performance (optimal use of double FPU) is: $2 \times 5.6$ GFLOPS $=11.2$ GFLOPS. A rack $(1,024$ nodes $=2,048 \mathrm{CPUs})$ therefore has 2.8 teraFLOPS or TFLOPS, and a peak of 5.6 TFLOPS. The Blue Brain Projects Blue Gene is a 4-rack system that has 4,096 nodes, equal to 8,192 CPUs, with a peak performance of 22.4 TFLOPS. A 64-rack machine should provide 180 TFLOPS, or 360 TFLOPS at peak performance.

\subsection{Modelling the Microcircuit}

The scheme shows the minimal essential building blocks required to recon- struct a neural microcircuit. Microcircuits are composed of neurons and synaptic connections. To model neurons, the three-dimensional morphology, ion channel composition, and distributions and electrical properties of the different types of neuron are required, are composed of neurons and synaptic connections. To model neurons, the three-dimensional morphology, ion channel composition, and distributions and electrical properties of the different types of neuron are required, as well as the total numbers of neurons in the microcircuit and the relative proportions of the different types of neuron. To model synaptic connections, the physiological and pharmacological properties of the different types of synapse that connect any two types of neuron are required, in addition to statistics on which part of the axonal arborization is used (presynaptic innervation pattern) to 
contact which regions of the target neuron (postsynaptic innervations pattern), how many synapses are involved in forming connections, and the connectivity statistics between any two types of neuron.

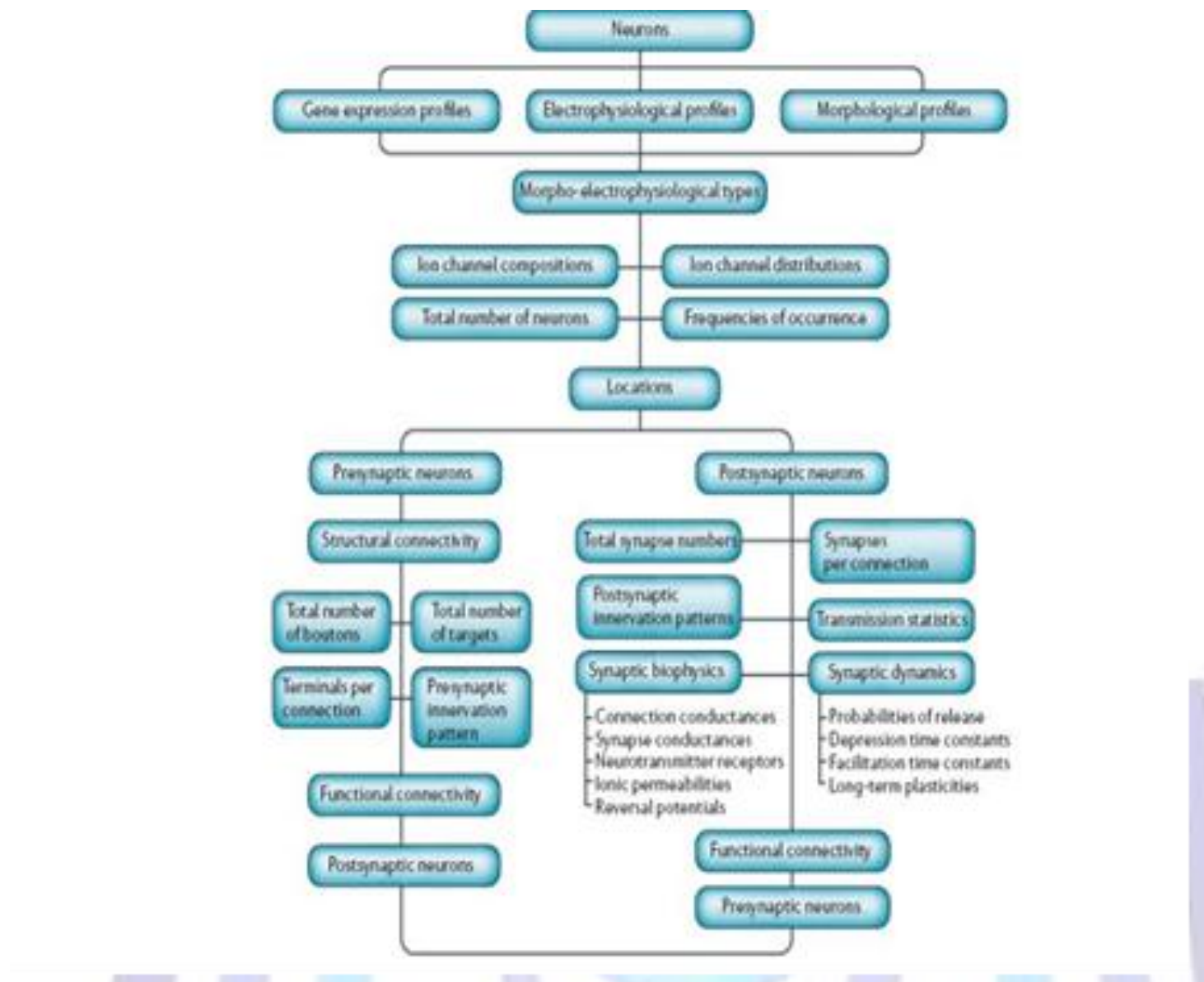

Fig. 1.2. Elementary building blocks of neural microcircuits.

Neurons receive inputs from thousands of other neurons, which are intricately mapped onto different branches of highly complex dendritic trees and require tens of thousands of compartments to accurately represent them. There is therefore a minimal size of a microcircuit and a minimal complexity of a neuron's morphology that can fully sustain a neuron. A massive increase in computational power is required to make this quantum leap - an increase that is provided by IBM's Blue Gene supercomputer. By exploiting the computing power of Blue Gene, the Blue Brain Project1 aims to build accurate models of the mammalian brain from first principles. The first phase of the project is to build a cellular-level (as opposed to a genetic- or molecular-level) model of a 2-week-old rat somatosensory neocortex corresponding to the dimensions of a neocortical column (NCC) as defined by the dendritic arborizations of the layer 5 pyramidal neurons. The combination of infrared differential interference microscopy in brain slices and the use of multi-neuron patch- clamping allowed the systematic quantification of the molecular, morphological and electrical properties of the different neurons and their synaptic pathways in a manner that would allow an accurate reconstruction of the column. Over the past 10 years, the laboratory has prepared for this reconstruction by developing the multi-neuron patch- clamp approach, recording from thousands of neocortical neurons and their synaptic connections, and developing quantitative approaches to allow a complete numerical breakdown of the elementary building blocks of the NCC. The recordings have mainly been in the 1416-day-old rat somatosensory cortex, which is a highly accessible region on which many researchers have converged following a series of pioneering studies driven by Bert Sakmann. Much of the raw data is located in our databases, but a major initiative is underway to make all these data freely available in a publicly accessible database. The so-called 'blue print' of the circuit, although not entirely complete, has reached a sufficient level of refinement to begin the reconstruction at the cellular level. Highly quantitative data are available for rats of this age, mainly because visualization of the tissue is optimal from a technical point of view. This age also provides an ideal template because it can serve as a starting point from which to study maturation and ageing of the NCC. As NCCs show a high degree of stereotypy, the region from which the template is built is not crucial, but a sensory region is preferred because these areas contain a prominent layer 4 with cells specialized to receive input to the neocortex from the thalamus; this will also be required for later calibration with in vivo experiments. The NCC should not be overly specialized, because this could make generalization to other neocortical regions difficult, but areas such as the barrel cortex do offer the advantage of highly controlled in vivo data for comparison. 
The mouse might have been the best species to begin with, because it offers a spectrum of molecular approaches with which to explore the circuit, but mouse neurons are small, which prevents the detailed dendritic recordings that are important for modelling the nonlinear properties of the complex dendritic trees of pyramidal cells $(75-80 \%$ of the neurons). The image shows the Microcircuit in various stages of reconstruction. Only a small fraction of reconstructed, three dimensional neurons is shown. Red indicates the dendritic and blue the axonal arborizations. The columnar structure illustrates the layer definition of the NCC.

- $\quad$ The microcircuits (from left to right) for layers 2, 3, 4 and 5.

- A single thick tufted layer 5 pyramidal neuron located within the column.

- One pyramidal neuron in layer 2, a small pyramidal neuron in layer 5 and the large thick tufted pyramidal neuron in layer

- An image of the NCC, with neurons located in layers 2 to 5.

\subsection{Simulating the Microcircuit}

Once the microcircuit is built, the exciting work of making the circuit function can begin. All the 8192 processors of the Blue Gene are pressed into service, in a massively parallel computation solving the complex mathematical equations that govern the electrical activity in each neuron when a stimulus $\square$ is applied. As the elec- trical impulse travels from neuron to neuron, the results are communicated via inter-processor communication (MPI). Currently, the time required to simulate the circuit is about two orders of magnitude larger than the actual biological time simulated. The Blue Brain team is working to streamline the computation so that the circuit can function in real time - meaning that 1 second of activity can be modeled in one second.

\subsection{Interpreting the Results}

Running the Blue Brain simulation generates huge amounts of data. Analyses of individual neurons must be repeated thousands of times. And analyses dealing with the network activity must deal with data that easily reaches hundreds of gigabytes per second of simulation. Using massively parallel computers the data can be analyzed where it is created

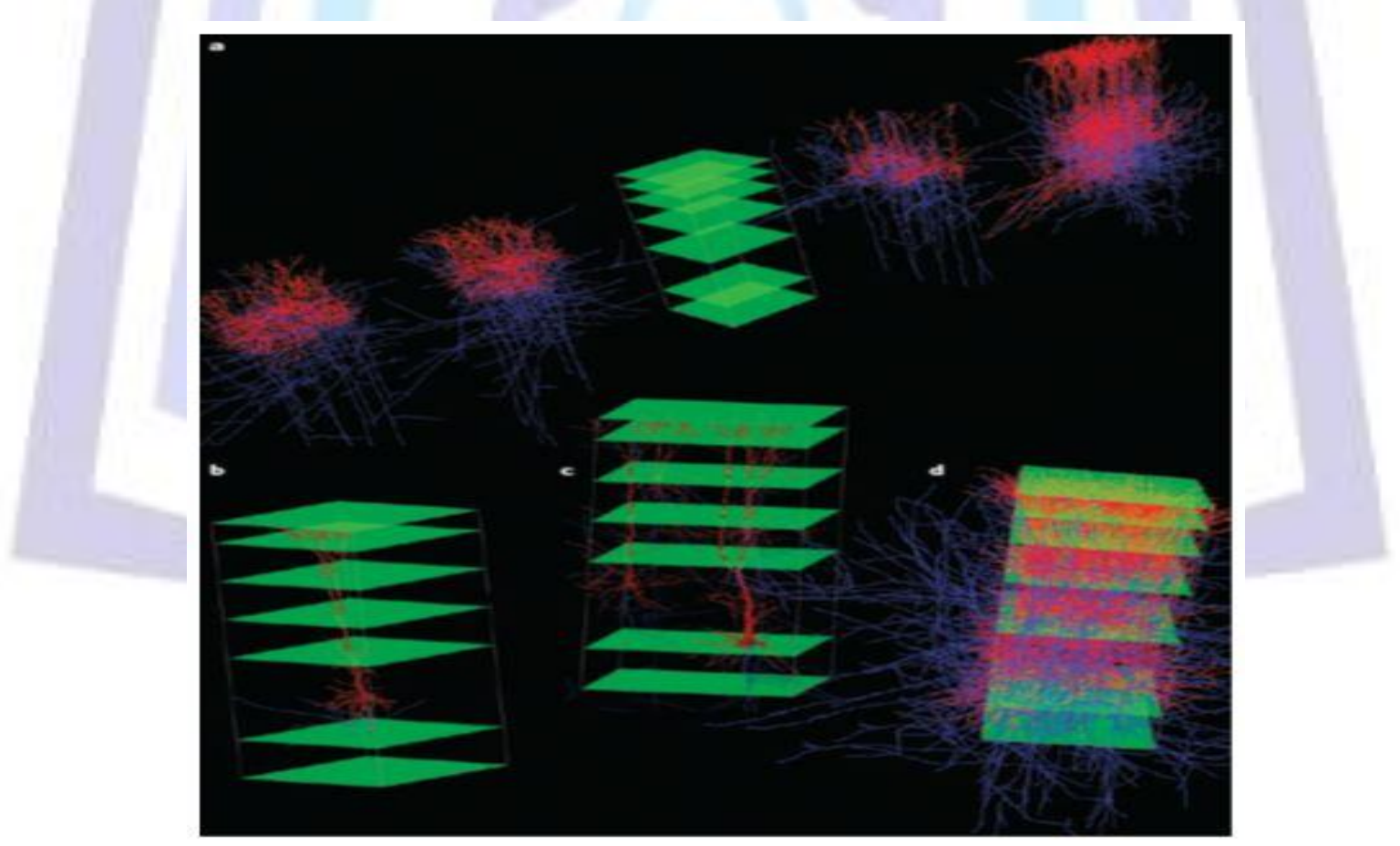

Fig. 1.3. Reconstructing the neocortical column.

(server-side analysis for experimental data, online analysis during simulation).Given the geometric complexity of the column, a visual exploration of the circuit is an important part of the analysis. Mapping the simulation data onto the morphology is invaluable for an immediate verification of single cell activity as well as network phenomena. Architects at EPFL have worked with the Blue Brain devel- opers to design a visualization interface that translates the Blue Gene data into a 3D visual representation of the column. A different supercomputer is used for this compu- tationally intensive task. The visualization of the neurons' shapes is a challenging task given the fact that a column of 10,000 neurons rendered in high quality mesh accounts for essentially 1 billion triangles for which about 100GB of management data is required. Simulation data with a resolution of electrical compartments for each neuron accounts for another 150GB. As the electrical 
impulse travels through the column, neurons light up and change color as they become electrically active. A visual interface makes it possible to quickly identify areas of interest that can then be studied more extensively using further simulations. A visual representation can also be used to compare the simulation results with experiments that show electrical activity in the brain

\subsection{Data Manipulation Cascade}

Building the Blue Column requires a series of data manipulations. The first step is to parse each three-dimensional morphology and correct errors due to the in vitro preparation and reconstruction. The repaired neurons are placed in a database from which statistics for the different anatomical classes of neurons are obtained. These statistics are used to clone an indefinite number of neurons in each class to capture the full morphological diversity. The next step is to take each neuron and insert ion channel models in order to produce the array of electrical types. The field has reached a sufficient stage of convergence to generate efforts to classify neurons, such as the Petilla Convention - a conference held in October 2005 on anatomical and electrical types of neocortical interneuron, established by the community. Single-cell gene expression studies of neocortical interneurons now provide detailed predictions of the specific combinations of more than 20 ion channel genes that underlie electrical diversity. A database of biologically accurate Hodgkin-Huxley ion channel models is being produced. The simulator NEURON is used with automated fitting algorithms running on Blue Gene to insert ion channels and adjust their parameters to capture the specific electrical properties of the different electrical types found in each anatomical class. The statistical variations within each electrical class are also used to generate subtle variations in discharge behaviour in each neuron. So, each neuron is morpho- logically and electrically unique. Rather than taking 10,000 days to fit each neuron's electrical behaviour with a unique profile, density and distribution of ion channels, applications are being prepared to use Blue Gene to carry out such a fit in a day. These functionalized neurons are stored in a database. The three-dimensional neurons are then imported into Blue Builder, a circuit builder that loads neurons into their layers according to a "recipe" of neuron numbers and proportions. A collision detection algorithm is run to determine the structural positioning of all axo-dendritic touches, and neurons are jittered and spun until the structural touches match experimentally derived statistics. Probabilities of connectivity between different types of neuron are used to determine which neurons are connected, and all axo-dendritic touches are converted into synaptic connections. The manner in which the axons map onto the dendrites between specific anatomical classes and the distribution of synapses received by a class of neurons are used to verify and fine-tune the biological accuracy of the synaptic mapping between neurons. It is therefore possible to place 10-50 million synapses in accurate three-dimensional space, distributed on the detailed threedimen- sional morphology of each neuron. The synapses are functionalized according to the synaptic parameters for different classes of synaptic connection within statistical vari- ations of each class, dynamic synaptic models are used to simulate transmission, and synaptic learning algorithms are introduced to allow plasticity. The distance from the cell body to each synapse is used to compute the axonal delay, and the circuit configuration is exported. The configuration file is read by a NEURON subroutine that calls up each neuron and effectively inserts the location and functional properties of every synapse on the axon, soma and dendrites. One neuron is then mapped onto each processor and the axonal delays are used to manage communication between neurons and processors. Effectively, processors are converted into neurons, and MPI (message-passing interface)- based communication cables are converted into axons interconnecting the neurons - so the entire Blue Gene is essentially converted into a neocortical microcircuit. We developed two software programs for simulating such large-scale networks with morphologically complex neurons. A new MPI version of NEURON has been adapted by Michael Hines to run on Blue Gene. The second simulator uses the MPI messaging component of the large-scale NeoCortical Simu- lator (NCS), which was developed by Philip Goodman, to manage the communication between NEURON-simulated neurons distributed on different processors. The latter simulator will allow embedding of a detailed NCC model into a simplified large-scale model of the whole brain. Both of these softwares have already been tested, produce identical results and can simulate tens of thousands of morphologically and electri- cally complex neurons (as many as 10,000 compartments per neuron with more than a dozen Hodgkin-Huxley ion channels per compartment). Up to 10 neurons can be mapped onto each processor to allow simulations of the NCC with as many as 100,000 neurons. Optimization of these algorithms could allow simulations to run at close to real time. The circuit configuration is also read by a graphic application, which renders the entire circuit in various levels of textured graphic formats. Real-time stereo visu- alization applications are programmed to run on the terabyte SMP (shared memory processor) Extreme series from SGI (Silicon Graphics, Inc.). The output from Blue Gene (any parameter of the model) can be fed directly into the SGI system to perform in silico imaging of the activity of the inner workings of the NCC. Eventually, the simulation of the NCC will also include the vasculature, as well as the glial network, to allow capture of neuron-glia interactions. Simulations of extracellular currents and field potentials, and the emergent electroencephalogram (EEG) activity will also be modelled.

\subsection{Whole Brain Simulations}

The main limitations for digital computers in the simulation of biological processes are the extreme temporal and spatial resolution demanded by some biological processes, and the limitations of the algorithms that are used to model biological 
processes. If each atomic collision is simulated, the most powerful super- computers still take days to simulate a microsecond of protein folding, so it is, of course, not possible to simulate complex biological systems at the atomic scale. However, models at higher levels, such as the molecular or cellular levels, can capture lower-level processes and allow complex large-scale simulations of biological processes. The Blue Brain Project's Blue Gene can simulate a NCC of up to 100,000 highly complex neurons at the cellular or as many as 100 million simple neurons (about the same number of neurons found in a mouse brain). However, simulating neurons embedded in microcircuits, microcircuits embedded in brain regions, and brain regions embedded in the whole brain as part of the process of understanding the emergence of complex behaviors of animals is an inevitable progression in understanding brain function and dysfunction, and the question is whether whole-brain simulations are at all possible. Computational power needs to increase about 1-millionfold before we will be able to simulate the human brain, with 100 billion neurons, at the same level of detail as the Blue Column. Algorithmic and simulation efficiency (which ensure that all possible FLOPS are exploited) could reduce this requirement by two to three orders of magnitude. Simulating the NCC could also act as a test-bed to refine algorithms required to simulate brain function, which can be used to produce field programmable gate array (FPGA)-based chips. FPGAs could increase computational speeds by as much as two orders of magnitude. The FPGAs could, in turn, provide the testing ground for the production of specialized NEURON solver application- specific integrated circuits (ASICs) that could further increase computational speed by another one to two orders of magnitude. It could therefore be possible, in principle, to simulate the human brain even with current technology. The computer industry is facing what is known as a discontinuity, with increasing processor speed leading to unacceptably high power consumption and heat production. This is pushing a qualita- tively new transition in the types of processor to be used in future computers. These advances in computing should begin to make genetic- and molecular-level simulations possible. Software applications and data manipulation required to model the brain with biological accuracy. Experimental results that provide the elementary building blocks of the microcircuit are stored in a database. Before three-dimensional neurons are modelled electrically, the morphology is parsed for errors, and for repair of arboriza- tions damaged during slice preparation. The morphological statistics for a class of neurons are used to clone multiple copies of neurons to generate the full morpho- logical diversity and the thousands of neurons required in the simulation. A spectrum of ion channels is inserted, and conductances and distributions are altered to fit the neurons electrical properties according to known statistical distributions, to capture the range of electrical classes and the uniqueness of each neurons behaviour (model fitting/electrical capture). A circuit builder is used to place neurons within a three- dimensional column, to perform axo-dendritic collisions and, using structural and functional statistics of synaptic connectivity, to convert a fraction of axo-dendritic touches into synapses. The circuit configuration is read by NEURON, which calls up each modelled neuron and inserts the several thousand synapses onto appropriate cellular locations. The circuit can be inserted into a brain region using the brain builder. An environment builder is used to set up the stimulus and recording conditions. Neurons are mapped onto processors, with integer numbers of neurons per processor. The output is visualized, analysed and/or fed into real-time algorithms for feedback stimulation.

\section{APPLICATIONS OF BLUE BRAIN PROJECT}

\subsection{What can we learn from Blue Brain?}

Detailed, biologically accurate brain simulations offer the opportunity to answer some fundamental questions about the brain that cannot be addressed with any current experimental or theoretical approaches. These include,

- Defining Functions of the Basic Elements

- Understanding Complexity

- Exploring the Role of Dendrites.

- Revealing Functional Diversity

- Tracking the Emergence of Intelligence

- Identifying Points ofVvulnerability.

- Simulating Disease and Developing Treatments

- Providing a Circuit Design Platform

\subsection{Applications of Blue Brain}

- $\quad$ Gathering and Testing 100 Years of Data

- Cracking the Neural Code

- Understanding Neocortical Information Processing

- A Novel Tool for Drug Discovery for Brain Disorders

- A Global Facility

- A Foundation for Whole Brain Simulations

- A Foundation for Molecular Modeling of Brain Function 


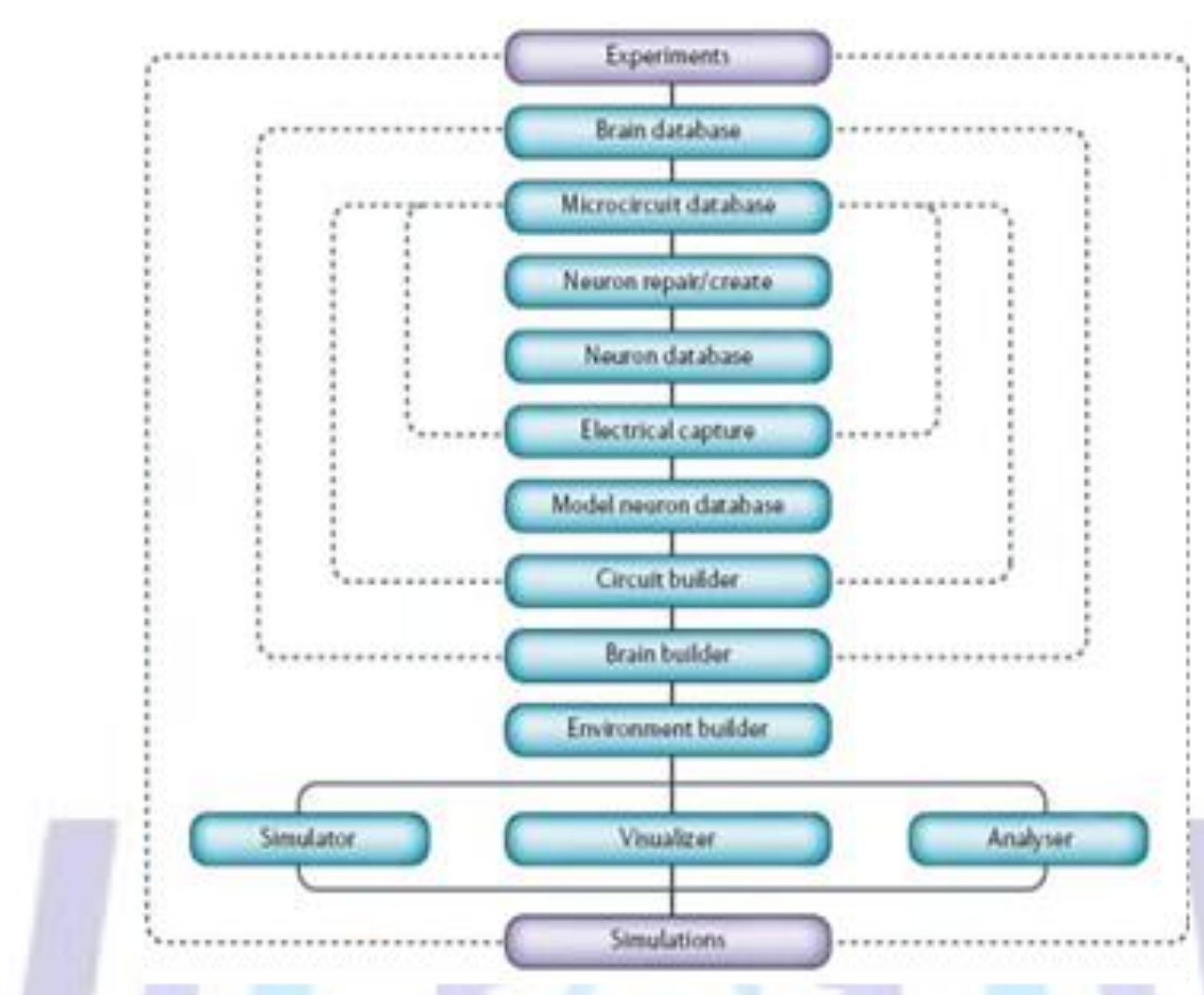

Fig. 1.4. The data manipulation cascade

\section{ADVANTAGES AND LIMITATIONS}

\subsection{Advantages}

- We can remember things without any effort.

- Decision can be made without the presence of a person.

- Even after the death of a man his intelligence can be used.

- The activity of different animals can be understood. That means by interpre- tation of the electric impulses from the brain of the animals, their thinking can be understood easily.

- It would allow the deaf to hear via direct nerve stimulation, and also be helpful for many psychological diseases. By down loading the contents of the brain that was uploaded into the computer, the man can get rid from the madness.

\subsection{Limitations}

Further, there are many new dangers these technologies will open. We will be susceptible to new forms of harm.

- We become dependent upon the computer systems.

- Others may use technical knowledge against us.

- Computer viruses will pose an increasingly critical threat.

- The real threat, however, is the fear that people will have of new technologies.

That fear may culminate in a large resistance. Clear evidence of this type of fear is found today with respect to human cloning.

\section{FUTURE PERSPECTIVE}

The synthesis era in neuroscience started with the launch of the Human Brain Project and is an inevitable phase triggered by a critical amount of fundamental data. The data set does not need to be complete before such a phase can begin. Indeed, it is essential to guide reductionist research into the deeper facets of brain structure and function. As a complement to experimental research, it offers rapid assessment of the probable effect of a new finding on preexisting knowledge, which can no longer be managed completely by any one researcher. Detailed models will probably become 
the final form of databases that are used to organize all knowledge of the brain and allow hypothesis testing, rapid diagnoses of brain malfunction, as well as development of treatments for neurological disorders. In short, we can hope to learn a great deal about brain function and disfunction from accurate models of the brain .The time taken to build detailed models of the brain depends on the level of detail that is captured. Indeed, the first version of the Blue Column, which has 10,000 neurons, has already been built and simulated; it is the refinement of the detailed properties and calibration of the circuit that takes time. A model of the entire brain at the cellular level will probably take the next decade. There is no fundamental obstacle to modeling the brain and it is therefore likely that we will have detailed models of mammalian brains, including that of man, in the near future. Even if overestimated by a decade or two, this is still just a 'blink of an eye' in relation to the evolution of human civilization. As with Deep Blue, Blue Brain will allow us to challenge the foundations of our understanding of intelligence and generate new theories of consciousness.

\section{CONCLUSION}

In conclusion, we will be able to transfer ourselves into computers at some point. Most arguments against this outcome are seemingly easy to circumvent. They are either simple minded, or simply require further time for technology to increase. The only serious threats raised are also overcome as we note the combination of biological and digital technologies.

\section{REFERENCES}

[1] "Engineering in Medicine and Biology Society", 2008. EMBS 2008. 30th Annual International Conference of the IEEE

[2] Henry Markram, "The Blue Brain Project", Nature Reviews Neuroscience 2006 February.

[3] Simulated brain closer to thought BBC News 22 April 2009.

[4] "ProjectMilestones".BlueBrain.http://bluebrain.epfl.ch/Jahia/site/bluebrain/op/edit/pid/19085

[5] Graham-Rowe, Duncan. "Mission to build a simulated brain begins", NewSci-entist, June 2005. pp. 1879-85.

[6] Special issue on brain-computer interface technology: The third international meeting. IEEE Transactions on Neural Systen Rehabilitation Engineering, 2006.

[7] E. Bart and S. Ullman. Cross-generalization: Learning novel classes from a single example by feature replacement. In CVPR, 2005.

[8] L. Fei-Fei, R. Fergus, and P. Perona. Learning generative visual models from few training examples: an incremental bayesian Approach Tested on 101 Object Cateories. In Workshop on Generative Model Based Vision, 2004.

[9] R. Fergus, P. Perona, and A. Zisserman. Object class recognition by unsupervised scale-invariant learning. In CVPR, 2003.

[10] B. Fisch. Fisch \& Spehlmann's EEG primer: Basic principles of digital and analog EEG. Elsevier: Amsterdam, 2005.

[11] A. Gerson, L. Parra, and P. Sajda. Cortically-coupled computer vision for rapid image search. IEEE Transactions on Neural Systems and Rehabilitation Engineering, 14(2):174-179, 2006.

[12] K. Grauman and T. Darrell. The pyramid match kernel: Discriminative classification with sets of image features. In ICCV, 2005.

[13] K. Grauman and T. Darrell. Approximate correspondences in high dimensions. In NIPS, 2007.

[14] K. Grill-Spector. The neural basis of object perception. Current opinion in neurobiology, 13:1-8, 2003.

[15] Y. Ivanov, T. Serre, and J. Bouvrie. Confidence weighted classifier combination for multi-modal human identification. Technical Report AI Memo 2005-035, MIT Computer Science and Artificial Intelligence Laboratory, 2005.

[16] Z. J., M. Marszalek, S. Lazebnik, and C. Schmid. Local features and kernels for classifcation of texture and object categories: A comprehensive study. IJCV, 2006.

\section{Author Biography}

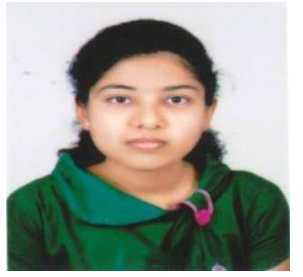

CHANDANI R. SURYAWANSHI holds Engg. degree in Electronics Engg. And doing M.E in WIRELESS COMMUNICATION AND COMPUTING from Rashtrasanta Tukdoji Maharaj Nagpur University, NAGPUR, INDIA 